\title{
Inhibition of LIM kinase inhibits cancer growth.
}

\author{
Juliana Antonipillai ${ }^{1 *}$, Sam L Francis ${ }^{2}$ \\ ${ }^{1}$ Western Centre for Health and Research Education, Victoria University, Australia \\ ${ }^{2}$ St Vincent's Hospital, The University of Melbourne, Australia
}

Accepted on 12 August, 2017

\section{Introduction}

The LIM kinase family of serine protein kinases contains two members, LIM kinase 1 (LIMK1) and LIM kinase 2 (LIMK2). LIMK1 was first isolated from olfactory epithelial cell lines [1], and LIMK2 from a rat cDNA library using human LIMK1 cDNA as a probe [2]. LIMK1 and 2 are structurally similar and share 50\% amino acid homology, human and mouse LIMK1 share $95 \%$ amino acid homology. The amino terminus of LIMK contains two double zinc finger LIM domains, followed by a PDZ domain. Both domains are involved in protein-protein interaction [3].

The C-terminus end of the molecule contains the catalytic active kinase domain. LIMK proteins are about $70 \mathrm{kDa}$ in size and are expressed in the cytoplasm of most adult and embryonic mouse tissues as well as human tissues [4]. Furthermore, the LIMK1 protein is significantly expressed in highly invasive breast and prostate cancer lineages compared to less invasive cell lines [5].

\section{Dynamics of LIMK and Actin}

$\mathrm{ADF} /$ cofilin, is a well-known substrate of the LIMK molecule, it acts as an actin depolymerization factor. LIMK interacts with to phosphorylate cofilin resulting in inactivation. Cofilin promotes actin depolymerization in the cell by hydrolysing actin filaments (F-actin) and sequestering the monomers (Gactin) from the polymerizing end (Figure 1).

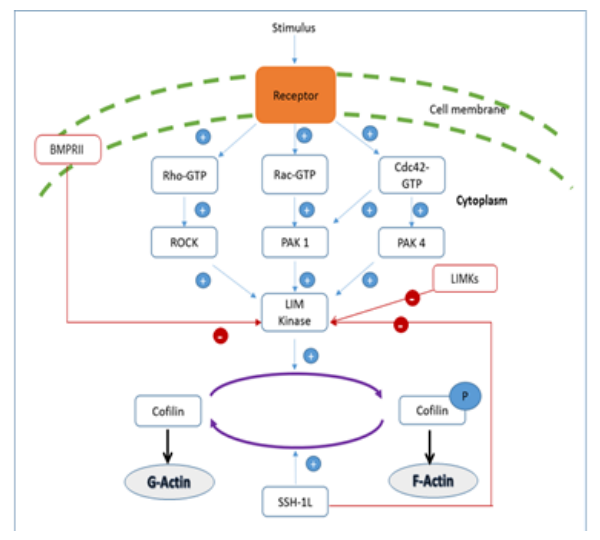

Figure 1. A model for LIMK signalling cascade in normal cells. LIMK activity is controlled by several molecules. While the Rho associated kinase (ROCK) and p21 activated kinases (PAKs) activate LIMK, Bone morphogenetic protein receptor II (BMPRII), dominantnegative form of LIMK (LIMKs) and Slingshot phosphatise (SSH-IL) inhibit its activity. Active LIMK promotes filamentous actin (F-actin) in cells by phosphorylating its substrate cofilin. But dephosphorylated LIMK is inactive and so promotes G-actin.
Phosphorylated cofilin looses its binding affinity toward the actin molecule leading to inhibition of the hydrolysis process, thus increasing polymerized filamental actin in the cells. Downstream Rho GTPases such as PAK1, PAK 4 and ROCK phosphorylate and activate LIMK molecules leading to activation of F-actin, whereas BMPRII, SSH-1L and the dominant form of LIMK, known as LIMKs, inactivate the molecule to promote G-actin. Coordinating together with other signaling molecules, the downstream LIMK signaling cascade is also involved in the homeostasis of actin dynamics under physiological conditions.

\section{LIMK and microtubule dynamics}

Microtubule (MT) destabilization promotes the formation of actin stress fibers and enhances the contractility of cells. Gorovoy et al. identified that LIMK1 is associated with MTs via their PDZ domain, and is involved in MT destabilization [6]. Moreover, phosphorylated LIM kinases (pLIMK1/2) are co-localized with gamma-tubulin in the centrosomes during the early mitotic phases of human breast and prostate cancer cells. In addition co-localization begins in the centrosomes during prophase and terminates during early telophase. However, accumulation of pLIMK $1 / 2$ at the cleavage furrow was noted. This observation suggests that activated LIMK1/2 may be associated with gamma-tubulin and play a role in mitotic spindle assembly [7].

\section{LIMK expression and cancer metastasis}

LIMK regulates the actin tredmilling process. Changes in actin polymerization are important features of motile cells. One of the hallmarks of metastatic cells is their ability to spread from the original site to other parts of the body 'metastasize'.

Cell motility is the result of coordination between membrane protrusive activity at a leading edge, movement of the cell body and retraction of the cell rear. Actin polymerization at the leading edge can physically drive membrane protrusion. Cofilin phosphorylation is essential for cell movement and since LIMK1 is the major cofilin kinase, it must be involved in cell movement $[8,9]$.

LIMK expression levels were elevated in metastatic cancer cells. We have demonstrated that the level of endogenous LIMK1 protein is markedly elevated in a large number of locally invasive breast and prostate cancer cell lines [10] as well as metastatic human tumours from breast, colon, skin and prostate. Bagheri-Yarmand et al. observed increased LIMK1 levels in metastatic breast cancer tissues. 
Their studies demonstrated that cells over expressing LIMK1 have increased levels of the serine protease urokinase type plasminogen activator (uPA) and its receptor (UPAR), furthermore they suggested that LIMK1 enhances tumour invasion by up-regulating the uPA system and inducing uPA secretion [10]. uPA is important for degradation of the extracellular matrix and facilitating cell motility.

\section{LIMK and therapeutic target}

LIMKs are an excellent target point for cancer therapy as they function to regulate the major intracellular network systems of microtubule dynamics and the actin cytoskeleton. Cell growth, invasion and migration were significantly reduced when kinase activity or gene expression of LIMKs were inhibited. Inhibition can be achieved via LIMK specific inhibitor molecules or via gene knockdown techniques, this has been shown in breast and lung cancer cells [11,12]. Recent research outcomes demonstrated that inhibition of LIMK activity blocks tumor progression not only via regulating actin dynamics but also by disrupting mitotic microtubule organization [13-16].

\section{References}

1. Bernard O, Ganiatsas S, Kannourakis G, et al. Kiz-1, a protein with LIM zinc finger and kinase domains, is expressed mainly in neurons. Cell Growth \& Differentiation. 1994;5:1159-71.

2. Nunoue $\mathrm{K}$, Ohashi K, Okano I, et al. LIMK-1 and LIMK-2, two members of a LIM motif-containing protein kinase family. Oncogene.1995;11:701-10.

3. Fanning AS, Anderson JM. Protein-protein interactions PDZ domain networks. Current Biology. 1996;6:1385-8.

4. Foletta VC, Moussi N, Sarmiere PD, et al. LIM kinase 1, a key regulator of actin dynamics, is widely expressed in embryonic and adult tissues. Exp Cell Res. 2004;294:392-405.

5. Yoshioka K, Foletta V, Bernard O, et al. A role for LIM kinase in cancer invasion. Proc Natl Acad Sci. 2003; 100:7247-52.

6. Gorovoy M, Niu J, Bernard O, et al. LIM kinase 1 coordinates microtubule stability and actin polymerization in human endothelial cells. J Biol Chem. 2005;280:26533-42.

7. Chakrabarti R, Jones JL, Oelschlager DK, et al. Phosphorylated LIM kinases colocalize with gammatubulin in centrosomes during early stages of mitosis. Cell Cycle. 2007;6:2944-52.
8. Worthylake R A, Burridge K, Rho A, et al. promote migration by limiting membrane protrusions. J Biol Chem. 2003;278:13578-84.

9. Wang W, Mouneimne G, Sidani M, et al. The activity status of cofilin is directly related to invasion, intravasation, and metastasis of mammary tumors. J Cell Biol. 2006;173:395-404.

10. Bagheri Yarmand, R Mazumdar, A Sahin, et al. LIM kinase 1 increases tumor metastasis of human breast cancer cells via regulation of the urokinase-type plasminogen activator system. Int $\mathrm{J}$ Cancer. 2006;118:2703-10.

11. Xia H, Sun S, Wang B, et al. miR-143 inhibits NSCLC cell growth and metastasis by targeting Limk1. Int J Mol Sci. 2014;15(7):11973-83.

12. Li R, Doherty J, Antonipillai J, et al. LIM kinase inhibition reduces breast cancer growth and invasiveness but systemic inhibition does not reduce metastasis in mice. Clin Exp Metastasis. 2013;30:483-95.

13. Prunier C, Josserand V, Vollaire J, et al. LIM Kinase Inhibitor Pyr1 Reduces the Growth and Metastatic Load of Breast Cancers. Cancer Res. 2016;76(12): 3541-52.

14. mardilovich $\mathrm{K}$, baugh $\mathrm{M}$, crighton $\mathrm{D}$, et al. LIM kinase inhibitors disrupt mitotic microtubule organization and impair tumor cell proliferation. Oncotarget. 2015;6(36): 38469-86.

15. prudent $\mathrm{R}$, vassal-Stermann $\mathrm{E}$, nguyen $\mathrm{CH}$, et al. Pharmacological inhibition of LIM kinase stabilizes microtubules and inhibits neoplastic growth. Cancer Res. 2012;72(17):4429-39.

16. Chen Q, Jiao D, Hu H, et al. Downregulation of LIMK1 level inhibits migration of lung cancer cells and enhances sensitivity to chemotherapy drugs. Oncol Res. 2013;20(11):491-8.

\section{*Correspondence to}

Juliana Antonipillai

Lecturer, ECDF,

School of Health and Biomedical Sciences,

RMIT University, PO Box 71,

Bundoora, Australia

E-mail: juliana.antonipillai@rmit.edu.au 